\title{
Im multimodalen Dialog. Zum Zusammenspiel von Text und Bild auf den Einbänden von Kinder- büchern
}

Tematem artykułu są teksty werbalno-ikoniczne, analizowane na przykładzie ilustrowanych okładek bajek dla dzieci, gdzie tytuł literacki wpisany jest w swoje wizualne otoczenie, stanowiąc jego integralną część. Relacje, w jakich pozostają wobec siebie tytuł bajki i jego wizualne otoczenie, są podstawą do tego, by okładki bajek dla dzieci traktować jako kompleksowe powierzchnie wizualne. W artykule prezentowane są liczne przykłady tego, jak tytuł bajki i środowisko graficzne wzajemnie się uzupełniają, pozostając w relacji partnerstwa i symbiozy. Artykuł stanowi także próbę odpowiedzi na pytanie, w jaki sposób kształtować takie powierzchnie wizualne na okładkach bajek, by dzięki nim skupić uwagę dziecka i zainteresować je lekturą bajki oraz równocześnie wspierać jego kompetencję językową.

Zum Thema des Beitrages werden Sprache-Bild-Texte, die auf bebilderten Kinderbucheinbänden auftauchen, bei denen der literarische Titel in seine visuelle Umgebung so eingebettet wird, dass er zu ihrem integralen Teil wird. Die Beziehungen, in denen der literarische Titel und seine visuelle Umgebung zueinander stehen, scheinen der Grund dafür zu sein, bebilderte Kinderbucheinbände als komplexe Sehflächen zu betrachten. Mit zahlreichen Abbildungen wird im Beitrag exemplifiziert, wie sich der literarische Titel und seine graphische Umgebung gegenseitig ergänzen, indem sie in Partnerschaft und Symbiose zueinander stehen. Der Beitrag gibt auch eine Antwort auf die Frage, wie Kinderbucheinbände als Sehflächen gestaltet werden sollten, um die Aufmerksamkeit der Kinder zu lenken, ihr Interesse am Buch zu erwecken und ihre sprachlichen Kompetenzen zu fördern.

The subject of the paper concerns verbal-iconic texts, analyzed on the example of the illustrated covers of fairy tales for children where the literary title is inscribed in its visual surroundings, simultaneously constituting its integral part. The relations in which the title of the fairy tale and its visual surroundings remain with the reference to 
Magdalena Makowska

one another, are a basis for treating covers of fairy tales for children as complex visual surfaces. The paper presents numerous examples of how the title of a given fairy tale and the graphic environment mutually complement each other, remaining in the relation of partnership and symbiosis. The article also constitutes an attempt at answering the question in what way to shape such visual surfaces on covers of fairy tales so as to, owing to them, focus a child's attention and make them interested in reading a particular fairy tale as well as simultaneously support their linguistic competence.

\section{Einleitung}

„Tatsächlich sind wir heute von Sehflächen aller Art umgeben, in denen Schrift und Bild durch ein beide Seiten verbindendes Design formal und inhaltlich untrennbar ineinander spielen" (SCHMITZ 2011a:4). Wenn die Frage danach gestellt wird, wie heutzutage kommuniziert wird, steht außer Zweifel, dass sich die rein sprachliche Kommunikation auf dem Rückzug befindet. STÖCKL (2004b:7) zufolge „[bildet] der rein sprachlich verfasste Text die Ausnahme und der multimodale Text gerät zur Norm, [...] die materialen Qualitäten von Sprache und ihre konkrete Medialität [rücken] in das Zentrum der Aufmerksamkeit - Sprachdesign oder Textdesign avanciert zu einem Schlüsselkonzept, das diese veränderte Gewichtung reflektiert“. Die rasante Technikentwicklung hat zweifelsohne dazu geführt, dass immer größere Informationsmengen verarbeitet werden. Gleichzeitig wird aber immer wieder nach Formen der Kommunikation gesucht, die den Rezipienten die Wahrnehmung von Informationen erleichtern könnten. Es wird nicht nur gesprochen und geschrieben, sondern auch gezeigt und vor Augen geführt, weil „die graphische Form [...] den ,Sinn“ eines ,Textes‘ ganz wesentlich mit[bestimmt]“ (SPITZMÜLlER 2009:459). Auch wenn in Anlehnung an LUDWIG JÄGER (2002:34) angenommen wird, dass Sprache immer noch ein starkes und flexibles Zeichenmedium ist und deshalb zum ,Archimedium‘ wird, lässt sich nicht mehr bestreiten, dass die Kommunikation nur selten durch ein Zeichensystem realisiert wird und eher als „eine integrative Verkopplung mehrerer Kodes“ (SCHNEIDER / STÖCKL 2011:14) betrachtet werden sollte. Das hat zur Folge, dass auch das wissenschaftliche Interesse an Texten, in denen verschiedene Zeichenressourcen synergetisch zusammenwirken, weiter zunimmt. Die multimodale Kommunikation, die in Anlehnung an ULRICH SCHMITZ (2011a:2) als „Verständigung über parallele Kanäle und mit mehreren Sinnen“ zu verstehen ist, hat ihren Ursprung darin, dass sich das Bildliche in der gegenwärtigen Kommunikationspraxis immer noch auf dem Vormarsch befindet und der Trend zu visueller Kommunikation immer 
stärker wird. Sowohl die sich etablierende linguistische Teildisziplin ,Bildlinguistik ${ }^{1}$ als auch Disziplinen wie Kultur- oder Literaturwissenschaft machen u.a. unterschiedliche Formen der verbal-bildlichen Synergie zu ihrem Gegenstand. Die einzelnen Elemente dieser sprachlich-bildlichen Partnerschaft können erst dann Bedeutung tragen, „wenn ihnen ein regelhafter Gebrauch zugeschrieben wird, wenn der Rezipient also glaubt, dass der Produzent ein bestimmtes gestalterisches Element intentional verwendet, um ihn (den Rezipienten) dadurch zu bestimmten Schlüssen zu verleiten“ (SPITZMÜLLER 2009:468). Es steht außer Zweifel, dass sowohl die Produktion als auch die Rezeption von Texten, in denen sprachliche und bildliche Elemente zusammenwirken, die Integration der verschiedenen Zeichenressourcen zu einer semantischen und funktionalen Einheit erfordert. Um mit solchen Texten umgehen zu können, braucht man eine bestimmte ,multimodale Kompetenz ${ }^{2}$, die sich auf kognitive und textpraktische Tätigkeiten bezieht (vgl. STÖCKL 2011:45). Hans-Jürgen Bucher weist darauf hin, dass die moderne Kommunikation, die durch zwei Entwicklungstendenzen, und zwar die Multimodalisierung und die Delinearisierung, gekennzeichnet ist, auf ganz bestimme Art und Weise den Rezipienten und seine Bedürfnisse berücksichtigt: „Die Medienkommunikation wird nicht nur über verschiedene Modi abgewickelt, sondern sie eröffnet dem Rezipienten auch die Freiheit, die Abfolge, in der die Angebote rezipiert werden, selbst zu bestimmen.“ (BUCHER 2011:125) Im Vertrauen auf seine Kompetenzen wird dem Rezipienten die Möglichkeit gegeben, Texte und ihre Botschaften für sich selbst zu ,dechiffrieren'.

$\mathrm{Zu}$ einer interessanten Verflechtung sprachlicher und bildlicher Ressourcen kommt es im Falle von Kinderbucheinbänden, die ein gutes Beispiel dafür sind, wie sprachliche und bildliche Elemente synergetisch zusammenwirken können. Der folgende Beitrag setzt sich zum Ziel, Einbände von Kinderbüchern als ,Sehflächen' (vgl. SCHMiTZ 2011, 2011a) zu beschreiben. Für dieses Kernthema werden folgende Schwerpunkte formuliert:

1 Nach KLemm / STÖCKL (2011:9) ist unter ,Bildlinguistik‘ nicht etwa eine ,Linguistik des Bildes' zu verstehen, sondern es handelt sich darum, die Bezüge zwischen Sprache und Bild in Gesamttexten zu betrachten. ,Bildlinguistik‘ nähert sich einer Sprache des Bildes oder einer Bildsprache.

2 Während Stöckl den Begriff der ,multimodalen Kompetenz‘ favorisiert, beschreibt JÄGER (2002:35) die Fähigkeit zur intra- und intermedialen Transkription als ,transkriptive Intelligenz'. Ausführlich über Komponenten multimodaler Kompetenz bei STÖCKL (2011). 
1. Ist es begründet, Bilder aus (text)linguistischer Sicht zu betrachten?

2. Welche Synergien und Schnittstellen ergeben sich aus sprachlichbildlicher Partnerschaft?

3. Welche kommunikativ-funktionalen Leistungen erbringen die Kinderbucheinbände als Sehflächen?

4. Wie werden die Titel von Kinderbüchern am häufigsten formuliert?

5. Ist es möglich, mit Hilfe von Kinderbucheinbänden den kindlichen Spracherwerb zu fördern?

Das Korpus bilden 100 Einbände von modernen deutschen Kinderbüchern. Die Auswahl des Korpus erfolgt unter Heranziehung sowohl technischer als auch quantitativer und qualitativer Merkmale. In technischer Hinsicht handelt es sich dabei um Texte, die das Internetportal Kinderbuch-Couch präsentiert, wobei der Zeitraum der berücksichtigten Kinderbücher die Jahre 2010 bis 2012 umfasst. $^{3}$ Diese Computerdatenbank wurde nach Schlagwörtern wie ,Kinderliteratur‘ / ,Kindermärchen“ abgefragt. In quantitativer Hinsicht werden nur Kinderbücher als Exemplare mit vergleichsweise großem Text- bzw. Titelumfang gewählt. In qualitativer Hinsicht werden Online-Ressourcen aufgenommen, die dank ihrem überregionalen Charakter ein breites Titelspektrum aufweisen.

\section{Ist es begründet, Bilder aus (text)linguistischer Sicht zu be- trachten?}

Die Frage danach, ob es begründet ist, Bilder aus linguistischer Sicht zu beschreiben, wird immer häufiger thematisiert. ${ }^{4}$ Sie hat ihren Ursprung darin, dass die Kommunikation nicht entweder mit Bildern oder rein sprachlichen Texten verläuft, sondern immer häufiger die Form von Text-Bild-Gemengen hat. Die eindeutige Bestimmung dessen, wo die Grenzen des sprachlichen Textes liegen und wo das reine Bild anfängt, wird immer komplizierter. Die beiden Zeichensysteme sind auf so vielfältige Weise miteinander verknüpft, dass ihre Botschaft als Ganze erst dank dem endlosen Wechselspiel zwischen Schrift und Bild entsteht. Die Tatsache, dass rein schriftsprachliche Texte

3 Alle im Beitrag verwendeten Abbildungen von Kinderbucheinbänden stammen von der Website: http://www.kinderbuch-couch.de/ (30.11.2012).

4 Dazu u.a. STÖcKL (2004, 2004a, 2011); Schmitz (2001, 2003a, 2004, 2005, 2011, 2011a); SPITZMÜLLER (2009); BuCHER (2011); Holly (2009); KRÄMER (2006); NÖTH (2000). 
immer seltener vorkommen, hat zur Folge, dass man nicht mehr „blind für Bilder“" (SCHMITZ 2005:189) sein darf. Schon 2001 hat UlLA FIX (2001:510) darauf hingewiesen, dass ,man [...] sich also in der Textlinguistik darauf [wird] einstellen müssen, dass der bildliche Text in ,gemischten' Texten gegenüber dem sprachlichen an Gewicht gewinnen wird, und Beschreibungsinventarien, die bisher nicht vorliegen, entwickelt [werden] müssen“. Mit diesen Worten verweist die Autorin darauf, dass es ,gemischte Texte` gibt, d.h. solche, in denen rein sprachliche und bildliche Elemente integriert werden. Die Bestätigung dafür findet man auch in neueren Beiträgen von Fix, in denen sie dafür plädiert, „dass der Textbegriff vom rein sprachlich bestimmten auf einen multikodalen erweitert werden muss“, denn „Texte existieren nie nur sprachlich, immer sind andere Zeichen an ihnen beteiligt, seien es Gestik, Mimik, Stimmführung oder - bei den uns interessierenden schriftlichen Äußerungen - Bilder, Typographie, Papiersorte, usw.“ (FIX 2008:31). Das stiftet die Hypothese, dass Bilder oder Typographie keine Randerscheinungen sind, sondern dass sie zu zentralen Elementen eines Textes werden, der als „eine formale, stilistische Einheit [gilt], in der alle Mittel zusammenwirken und in der sich insofern ein Mittel aus dem anderen erklärt“ (FIX 2009:128). Auch wenn nicht direkt festgestellt wird, dass Bilder Texte sind, fungieren sie mindestens als bedeutungstragende Textteile. Eine ähnliche Meinung vertritt ERNEST HESS-LÜTTICH (1997:132), indem er numerischen Tabellen, graphischen Abbildungen, Fotos oder eben Bildern den Status nicht-verbaler Texte zuschreibt. Im Kontext der Hypermedien spricht ANGELIKA STORRER (2004:55) über „Text-Bild-Texte“, was eindeutig besagt, dass neben sprachlichen auch bildliche Teiltexte vorkommen. NORBERT WOLF (2000:289) zufolge werden „Texte - denn es sind ja immer Texte, die realisiert und somit auch verschriftlicht werden - [...] zuvörderst mit den Augen wahr- und aufgenommen; Texte wirken mithin wie Bilder, wenn nicht gar als Bilder“. Für Hans-Jürgen Bucher steht es außer Zweifel, dass sprachliche Texte dank Bildern visuell informativer werden, was der Grund dafür ist, sprachliche Texte und Abbildungen derselben Erschließungsebene, und zwar der Informationsebene, zuzuschreiben. Die zweite, d.h. operationale Ebene, bilden dagegen u. a. Typographie oder Textdesign. Während auf der Informationsebene das Wissen in Text und Bild verfügbar ist, ermöglicht die operationale Ebene die Erschließung des Wissens- und Informationsangebotes (vgl. BuCHER 2000:675). Der Autor plädiert zwar nicht dafür, Bilder als Texte zu beschreiben, aber er betont, dass Bilder informativ sind und die Informativität von sprachlichen Texten wesentlich heben können. BUCHER (2011:123) zufolge sind „Bild und Text [...] nur die prominentesten Vertreter einer Vielfalt 
von Kommunikationsmodi wie Design, Typographie, Farben, Grafiken, Piktogramme oder operationale Zeichen“, die „[...] zusammen eine komplexere Kommunikationsform bilden“. Auch Winfried Nöth unterscheidet eindeutig zwischen Texten und Bildern, wobei er sich in seiner Analyse auf statische (stehende) Bilder im Kontext von schriftlichen Texten und geschriebene oder gedruckte Texte beschränkt. Der Autor stellt Fragen nach Gemeinsamkeiten und Unterschieden zwischen Texten und Bildern, indem er sowohl ihre kognitive Verarbeitung analysiert, als auch auf ihre semiotische Struktur Rücksicht nimmt. Aus der Analyse der intermedialen Text-Bild-Beziehungen zieht er u.a. folgende Schlussfolgerung: „Zwischen Bild und Text finden Transformationen und Substitutionen statt: aus Bildern werden Texte, aus Texten Bilder.“ (NÖTH 2000:495)

Eine besonders interessante Diskussion darüber, über welchen Status Bilder verfügen, führen Stöckl und Schmitz. STÖCKL (2004:103) bevorzugt die These, dass Bilder als der Sprache gleichrangige und mit ihr vernetzte Zeichenobjekte zu betrachten sind. Der Autor unterscheidet dabei zwischen sprachlichen und bildlichen Textteilen, weist auf ihren wechselseitigen Bezug hin und ist überzeugt, dass ihr Bedeutungsspiel nicht nur offene Interpretationen zulässt, sondern auch das Kommunikationsdesign reflektiert und dem Rezipienten eine aktive Sinnstiftung ermöglicht. STÖCKL (2004a:17) zufolge gelten Schrift und Bild als ,mediale Varianten', die sich je einer ,zentralen Zeichenmodalität‘ zuordnen lassen. Während die Schrift eine mediale Variante der Zeichenmodalität Sprache ist, die auch die gesprochene Sprache umfasst, gilt das statische Bild als eine mediale Variante der Zeichenmodalität Bild, zu der auch bewegte Bilder gehören. Wenn Stöckl über Botschaften spricht, in denen sprachliche und bildliche Ressourcen vorkommen, dann steht es für ihn außer Zweifel, dass man es mit Gesamttexten zu tun hat und Bilder als ihre wertvollen Teile fungieren. Der Autor betont, dass „die bildlich kodierten Teiltexte für die wesentlichen Textbeschreibungsebenen relevant sind und konstitutive Bestandteile der Textstrukturierung ausmachen“ (STÖCKL 2004:108). Das findet seine Bestätigung in der von Stöckl gebrauchten Terminologie: Der Autor spricht konsequent von Sprache-Bild-Texten, d.h. von Gesamtbotschaften, die als Resultat dessen zu betrachten sind, dass sprachliche und bildliche Textteile zusammenwirken. Das hat zur Folge, dass sie als Ganze über den Textstatus verfügen. Das zeigt aber auch eindeutig, dass Stöckl den Bildern den Status von Textteilen zuschreibt und für die Gliederung des Gesamttextes in Bild- und Sprachteil plädiert. Der Autor weist auch darauf hin, dass „,der multimodale Text [...] als der Normalfall des Kommunizierens, und nicht als ein überkomplexer Sonderfall [erscheint]“ (STÖCKL 
2011:47). Stöckl geht davon aus, dass „Textverstehen immer als integrative und interdependente Kopplung aus verbalen und visuellen Verstehensprozessen zu betrachten [ist]“, wobei „an der Schnittstelle der beiden Codes [...] ein semantischer ,Spielraum ‘ [entsteht], der vielfältige Möglichkeiten der Bedeutungskonstruktion eröffnet“ (STÖCKL 2005:71). Im Fall von Sprache-BildTexten kommt es nach STÖCKL (2005:64) zu der Situation, dass der Bildteil seinen Rezipienten ein Bedeutungspotenzial gibt, das durch einen Kontext aktiviert und erschlossen werden muss. Diesen Kontext sichert der sprachliche Begleittext. Klemm und Stöckl sind sich auch dessen bewusst, dass die Linguisten dem Bild mit Skepsis begegnen und sich zu sehr auf die rein sprachliche Kommunikation konzentrieren. Trotzdem plädieren beide Autoren für die ,Bildlinguistik', verstanden als die Betrachtung der Bezüge, zu denen es zwischen Sprache und Bild in Gesamttexten kommt (vgl. KLEMM / STÖCKL 2011:9). Dieses Plädoyer hat seinen Ursprung darin, „dass man ,Sprache pur' (Holly 2009:389) in der kommunikativen Realität und in der textanalytischen Praxis genauso wenig haben kann wie ,Bilder pur““ (KLEMM / STÖCKL 2011:10). Den Ausdruck seiner Überzeugung, dass Bilder über potenzielle Mehrdeutigkeit und funktionale Polyvalenz verfügen, gibt Stöckl u. a. dadurch, dass er für ,visuelle Textualität` plädiert und vorschlägt, Bilder unter Berücksichtigung der Textualitätskriterien von DE BEAUGRANDE / DRESSLER (1981) zu beschreiben. Nach STÖCKL (2004:102) lassen sich Bilder mit Hilfe von ähnlichen Kriterien wie sprachliche Texte beschreiben, weil ihre Zeichenkomplexe den Regeln der räumlichen Grammatik folgen.

Im Unterschied zu Stöckl vertritt EHLICH (2005:59) die Meinung, dass Bilder keine Texte sind. Der Autor geht davon aus, dass sich sowohl geschriebene Texte als auch Bilder der Visualität bedienen und als solche ,gelesen' werden. Schriftliche Texte bilden aber nur eine Teilmenge von möglichen Texten. Ehlich zufolge entfalten sowohl Bilder als auch Texte semantische Potenziale, aber diese sind Potenziale je eigener Art. Der von Ehlich thematisierte Bezug auf die Visualität, der Bildern und schriftlichen Texten gemeinsam ist, führt KRÄMER (2006:79) zu der Feststellung, dass selbst die Schrift, die der Materialität des Textes zugrunde liegt, als eine Hybride aus Sprache und Bild betrachtet werden kann. Daraus zieht die Autorin die Schlussfolgerung, dass geschriebene Texte „,...] nicht einfach ,Texte‘ [bilden], sondern zuerst einmal eine ,Textur' [sind]: ein Gewebe von räumlichen Relationen“ (KRÄMER 2006:79).

Eine Bestätigung dafür, dass schriftliche Texte mehr als nur die Schrift in der Fläche sind, findet man auch bei ScHMITZ (2011a:3). Zu seinem Forschungs- 
gegenstand werden dabei Text-Bild-Verknüpfungen, d.h. Gefüge, in denen im Unterschied zu Sprache-Bild-Texten im Sinne von Stöckl - nur rein sprachliche Botschaften über den Textstatus verfügen. Schmitz zufolge entfalten solche Verknüpfungen sog. Sehflächen, d. h. „Flächen, auf denen Texte und Bilder in geplantem Layout gemeinsame Bedeutungseinheiten bilden“. Im Alltag gibt es immer häufiger Situationen, in denen solche sprachlichbildlichen Konglomerate ihre Anwendung finden. ,Pure“ Texte, d.h. rein sprachliche Texte, scheinen eigentlich nur für bestimmte literarische Gattungen ,reserviert‘ zu sein. Auch wenn ein verbaler Text ohne seine bildliche Begleitung erscheint, gewinnt seine visuelle Form immer mehr an Bedeutung, weil im Falle von verbalen Texten nicht nur ihr Inhalt, sondern auch ihre Form etwas zum Ausdruck bringt, semantisch relevant wird und funktional-kommunikativ wirkt, weil „Schrift [...] immer ein Schriftbild in der Fläche [erzeugt]" (Schmitz 2003:617). Nicht nur die inhaltliche Ebene des sprachlichen Textes, sondern auch die Art und Weise, wie dieser Text kommuniziert wird, ist für den Rezipienten relevant, weil das die Lektüre des Textes wesentlich mitbestimmt.

\section{Welche Synergien und Schnittstellen ergeben sich aus sprach- lich-bildlicher Partnerschaft?}

Es steht außer Zweifel, dass die Wahrnehmung von sprachlich-bildlichen Gefügen anders als im Falle von rein sprachlichen Texten verläuft. Bilder, die ihrer Natur nach zweidimensional sind, werden holistisch, simultan wahrgenommen (vgl. NÖTH 2000:490). Da sie ihre Rezipienten stärker aktivieren, werden sie auch schneller als sprachliche Texte aufgenommen. Diese höhere Aktivierung führt dazu, dass das langfristige Erinnern stimuliert wird und Bilder besser als Texte erinnert werden. In welcher Reihenfolge ein Rezipient einzelne Bildelemente betrachtet, hängt von ihm ab. Er kann seine Aufmerksamkeit beliebig führen und seine Wahrnehmung wird dadurch nicht gestört, was vor allem bei Kindern als Rezipienten besonders relevant ist. BUCHER (1996:48) weist darauf hin, dass „der Einstieg in die Seite immer über das Bild [erfolgt], wobei Farbbilder Schwarz-Weiß-Bilder normalerweise dominieren“. Die Bestätigung dieser These findet man auch bei BuRGER (2005:401), der überzeugt ist, dass in manchen Situationen Sprache und Bild asynchron zueinander sind. Im Falle von Sprache-Bild-Gefügen lassen sich Sprache und Bild nicht dem gleichen Zeitpunkt zuschreiben: Das Bild wird als erstes wahrgenommen, erst dann wird die Schrift rezipiert. Auch 
JANICH (2005:60) ist überzeugt, dass Bilder notwendig sind, „wenn Aufmerksamkeit erregt, emotionale Inhalte vermittelt und Produkte präsentiert werden sollen“. Demgegenüber werden Texte linear produziert und sukzessiv rezipiert (vgl. NÖTH 2000:490), also „von Teilen ausgehend zu einem Ganzen hin erlesen“ (SCHMITZ 2004:68). Schmitz weist aber auch darauf hin, dass „,...] Textlektüre heute [...] einen optisch orientierten ganzheitlichen Zugang von außen nach innen [pflegt]: Ähnlich wie bei der Lektüre von Bildern wird zuerst das visuelle Erscheinungsbild als Ganzes erfasst.“ (SCHмITZ 2003a:248) Es ist natürlich möglich, schriftsprachliche Texte auch dann zu verstehen, wenn nur einzelne Passagen gelesen und die anderen nur übersprungen werden. Nach EHLICH (2005:58) kann man aber den ganzen Text erst dann verstehen, wenn man der Linearität dieses Textes folgt. Gefüge, in denen sowohl sprachliche als auch bildliche Botschaften übermittelt werden, ,weichen den hergebrachten Unterschied zwischen linearer (sequenzieller) Textlektüre und ganzheitlicher (simultaner) Bildwahrnehmung zunehmend auf“" (SCHMITZ 2003:611). SCHMITZ (2011:104) macht auch darauf aufmerksam, dass „Bilder allein [...] Blicke fangen können, aber kaum abstrakte Botschaften übermitteln, nicht einmal Produkteigenschaften erläutern, geschweige denn Stoffnamen, Gattungsbezeichnungen oder auch nur Eigennamen (z.B. Markennamen) nennen“. Bild und Schrift ergänzen einander, sie gelten als ,Blickfang und Mitteilung', als Hybride aus Sprache und Bild, in der „Sprachliches und Bildliches sich verschwistern“ (KRÄMER 2006:81).

\section{Welche kommunikativ-funktionalen Leistungen erbringen die Kinderbucheinbände als Sehflächen?}

Als Beweis dafür, dass die klassische Polarisierung von Text und Bild aufgegeben wird und vielfältige Metamorphosen und Übergänge zwischen den beiden Ressourcen möglich werden, gelten Kinderbucheinbände. Es scheint begründet zu sein, diese Einbände als Sehflächen zu betrachten, deren verbale und bildliche Elemente synergetisch zusammenwirken. Diese stören einander nicht, sondern unterstützen sich gegenseitig und sind analytisch kaum zu trennen. Dank dieser sprachlich-bildlichen Verknüpfung generieren die Kinderbucheinbände Aufmerksamkeit und erzeugen emotions- und konnotationsgeladene Anmutungen. Es steht außer Zweifel, dass jeder verwendete 
Code bzw. jede gebrauchte Modalität ${ }^{5}$ grundverschieden ist und in einem solchen Text-Bild-Gefüge spezifische Aufgaben übernehmen kann. Das lässt sich im Falle von Sehflächen wie Kinderbucheinbänden ganz gut beobachten, weil sie die räumlichen und funktionalen Grenzen zwischen dem, was sprachlich, und dem, was graphisch ist, auflösen. Selbst wenn angenommen wird, dass eine solche Sehfläche aus einzelnen Teilflächen besteht, d.h. aus einer Bildfläche und einer Schriftfläche, dann lässt sich auch feststellen, dass die beiden Teilflächen „einander kontextualisieren und monosemieren“ (vgl. SCHMITZ 2003a:244). Auf solchen Einbänden wandert Bild in Text so ein, dass die beiden auf vielfältigste Weise in Beziehung gesetzt und gemischt werden. Dadurch entstehen verschiedene Zusammenhänge, die solche Einbände zu echten Sehflächen machen. Diese Zusammenhänge zwischen den sprachlichen und bildlichen Elementen einer Sehfläche werden u.a. dank bestimmten Kohäsionsmitteln möglich.

Abb. 1: Barth, Rolf: Herr Wolke und der 1. FC Toby

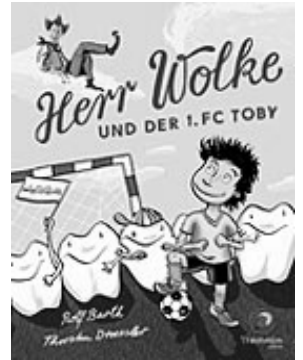

Abb. 2: Horacek, Petr: Greta Gans

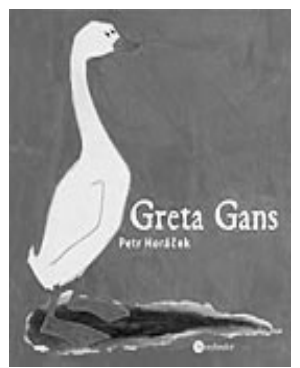

In Abb. 1 lassen sich mindestens zwei Kohäsionsmittel feststellen. Das erste ist die Blickrichtung der abgebildeten Person, die der Teilfläche ,Bild` zuzuschreiben ist. Der Blick geht aber nicht nur in den Titel hinein, sondern auch in seine direkte Nähe, wo sich der im Titel genannte Herr Wolke befindet. So entsteht eine Art der ,semantischen Kette': Die abgebildete Person schaut auf den auf der Wolke sitzenden Herrn, dessen Name in dem in der Nähe platzierten Titel wiederkehrt.

5 Vgl. StÖCKL (2004:102). Immer noch werden Begriffe wie ,Code“ oder ,Mode“ nicht einheitlich verwendet. Während z.B. Holly (2009:392) ,Codes‘ (z.B. verbale Zeichen) als materielle Zeichenqualitäten und ,Modes‘ als Wahrnehmungsqualitäten (z. B. auditiv, visuell) betrachtet, akzeptieren Autoren wie z.B. Stöckl diese Unterscheidung nicht und verstehen unter ,Modes‘ Zeichen jeglicher Art im Kommunikationsprozess. 
Als kohäsionsstiftendes Element einer Sehfläche, die auf einem Kinderbucheinband entsteht, kann auch die gebrauchte Farbe betrachtet werden. Die in Abb. 2 dargestellte Gans ist weiß, d.h. genauso wie die Farbe der im Titel gebrauchten Schrift. So fungiert die weiße Farbe als kohäsionsstiftendes Element im Rahmen einer Sehfläche. Interessant ist aber nicht nur die im Titel gebrauchte Farbe, sondern auch die Art und Weise, wie der Titel formuliert wird. Er lautet nicht Gans Greta (Appellativum+Eigenname), sondern eben Greta Gans (Eigenname+Eigenname), was die Gans noch zusätzlich personifiziert. Auch diese räumliche Nähe, die zwischen der Abbildung der Gans und deren Namen festzustellen ist, kann als kohäsionsstiftendes Element interpretiert werden.

Abb. 3: Schmidt, Hans-Christian / Walentowitz, Steffen: Töff Töff Töff Wir fahren Eisenbahn!

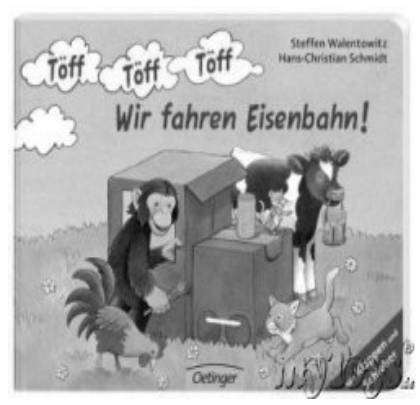

Abb. 4: Grosche, Erwin: E-le-fa, Ele-fee! Was macht der Elefant am See?

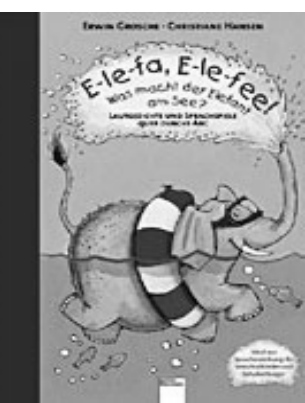

SANDIG (2000:10) zufolge „[interpretieren wir] aufgrund unserer kulturbedingten Sehgewohnheiten (Kress/van Leeuwen 1996) auch räumliche Nähe auf einem Textträger als Zeichen der Zusammengehörigkeit“. Diese Art der Zusammengehörigkeit kommt u.a. dann zum Ausdruck, wenn im Rahmen der Sehfläche sprachliche Botschaften als Sprech- oder Denkblasen erscheinen (Abb. 3-4). In Abb. 3 kommt es zu einer solchen Situation: Die sprachlichen Elemente erscheinen nicht nur im Rahmen der Schriftfläche als Titel Wir fahren Eisenbahn, sondern sie sind auch in die visuelle Umgebung dieses Titels eingebettet (Drei-Töff-Wolken). Den Hauptteil der in Abb. 4 präsentierten Sehfläche bildet ein Elefant, an dessen Rüssel eine Sprechblase auftaucht, in der der Titel dieses Buches erscheint.

Die Zusammengehörigkeit der Elemente, die eine gemeinsame Sehfläche bilden, kommt aber auch dadurch zum Ausdruck, dass diese Elemente kohärent sind. Nach STÖCKL (1997:143) kommt die Kohärenz in Sprache-BildTexten immer dann zustande, wenn „visuelle Zeichenkomplexe auf eine 
bestimmte Art und Weise auf den Text verweisen. Oder umgekehrt: Formulierungen des Texts nehmen gezielt auf das Bild Bezug. Der Text baut bewusst semantische Brücken zum Bild.“ SCHMITZ (2001:148) weist auch darauf hin, dass im Falle von Text-Bild-Gefügen die Kohärenz „[...] nicht mehr ,selbst-verständlich‘ im Text vermutet wird, sondern [...] aktiv aus Spannungen zwischen Text und Bild erst hergestellt werden [will]“. SCHOLz (1998:105) zufolge kommt in solchen Situationen die sog. Bildkompetenz zur Sprache. Es muss aber betont werden, dass diese Kompetenz in der Sozialisierung in enger Kopplung mit dem Spracherwerb erworben wird. Nach STÖCKL (2004a:92) umfasst sie „im weitesten Sinne ,das Lesen“, aber auch die kritische Beurteilung und das produktive Herstellen und Gestalten bzw. reproduktive Verwenden bildhafter Symbolsysteme“.

Abb. 5: Joslin, Mary: Der vierte König

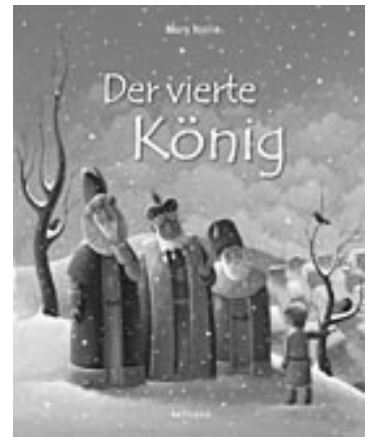

Ein sehr interessantes Beispiel dafür, wie die Kohärenz auf Kinderbucheinbänden realisiert werden kann, ist die Abb. 5. Als ,Einstieg‘ in diese Sehfläche fungiert das in ihrem Zentrum erscheinende Bild, auf dem drei Könige zu sehen sind. Dann wird das sprachliche Element dieser Sehfläche wahrgenommen, und zwar der Titel Der vierte König. Mikrotypographisch betrachtet scheint nur ein Element dieses Titels, und zwar das Substantiv König, hervorgehoben zu sein. Erst dank der Lektüre des Titels wird klar, dass eben der vierte König, der auf der Sehfläche kaum zu sehen ist, zur wirklichen Hauptfigur wird. So weist der Titel auf das Bild hin, auf dem der Rezipient die rechts stehende Person des vierten, kleinen Königs finden kann. 
Abb. 6: Nöstlinger, Christine: Guter Drache und böser Drache

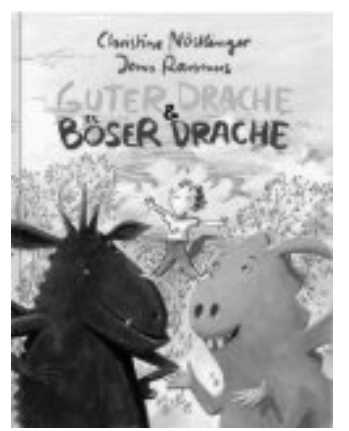

Kohärenzstiftend wirken auch die Elemente der Sehfläche Guter Drache und böser Drache von Christine Nöstlinger (Abb. 6). Die Kohärenz kommt in diesem Fall dadurch zum Ausdruck, dass eine Art Isotopiekette (vgl. GREIMAS 1971) gebildet wird. Diese Kette kommt sowohl auf der sprachlichen (antonymische Opposition im Titel: gut - böse) als auch auf der visuellen Teilfläche (das ,visuelle‘ Gegeneinander von zwei Drachen) zustande. Die so verstandene Kohärenz wird auch dadurch verstärkt, dass im Falle dieser Sehfläche die Farbe zu einem Mittel wird, das die Schriftfläche mit der Bildfläche visuell verknüpft. Dank diesem Element der mikrotypografischen Ebene erfährt der Rezipient noch vor der Lektüre des Textes, wie die beiden Drachen aussehen und welcher von ihnen gut (grüne Farbe) bzw. böse (rote Farbe) ist.

Vor allem die typographische Ebene scheint zu einem konstitutiven Merkmal von Sehflächen zu werden. STÖCKL (2004b:15) zufolge ist die Typographie „der Körper des Textes, seine unverzichtbare Lebensgrundlage, der Ort und der Stoff seiner Existenz“. Neben der Form des Textes umfasst sie auch die Auswahl und Verwendung von Schrift im Text sowie die gesamte graphische Gestaltung eines Schriftstücks oder Dokuments (STÖCKL 2004b:5). Eben in diesem Sinne wird die Typographie zu einem Merkmal von sprachlichbildlichen Gefügen, das sie zu Sehflächen macht und ihre Oberfläche strukturiert. STÖCKL (2004b:16) weist darauf hin, dass es legitim ist, die so verstandene Typographie als ,periphere Modalität` zu beschreiben und sie als solche von den zentralen Zeichenmodalitäten (Sprache, Bild) zu unterscheiden. Der Autor weist dabei auf drei Arten der Typographie hin. Neben der Mikrotypographie, die vor allem Gestaltungsdimensionen wie Schriftart, Schriftgröße, Schriftschnitt und Schriftfarbe umfasst, unterscheidet er auch zwischen der 
Mesotypographie, die sich auf die Gestaltung des Schriftbilds in der Fläche bezieht (z. B. Zeichen- und Wortabstand, Textmenge auf der Seite, Schriftmischungen), und der Makrotypographie, die vor allem die Organisation des Textes, d.h. Gliederung, Infoverteilung oder visuelle Akzentsetzung fokussiert (vgl. STÖCKL 2004b:22).

Mit der Frage der Textoberfläche beschäftigt sich auch Spitzmüller. In seinen Beiträgen beweist er eindeutig, dass die materielle Oberfläche des Textes als semiotische Ressource betrachtet werden kann. SPITZMÜLlER (2009:466) stellt die These auf, „dass sich die Bedeutung eines Textes in der Regel nicht nur aus einer einzelnen Zeichenressource (also etwa verbaler Sprache) konstituiert, sondern dass zumeist mehrere Zeichenressourcen (Modalitäten) zusammenwirken und damit das Interpretationsangebot eines Textes formieren“. Diese Elemente der Textoberfläche sind auch dadurch gekennzeichnet, dass sie nicht nur die ganze Sehfläche organisieren, sondern dass sie dabei kulturelles Wissen evozieren (vgl. SPITZMÜLLER 2009:464). Die Texte, die zu Sehflächen werden, werden so gestaltet, dass sie für die Mitglieder eines Kulturkreises verständlich sind, indem sie an die in diesem Kulturkreis gebrauchten Sprache oder Bildzeichen anknüpfen. Das scheint im Fall von Kindern als Rezipienten besonders relevant zu sein. Der Umgang mit solchen Sehflächen macht nicht nur Spaß, sondern er gibt auch die Möglichkeit, etwas zu lernen.

Abb. 7: Carls, Claudia: Woher kommt der Wind?

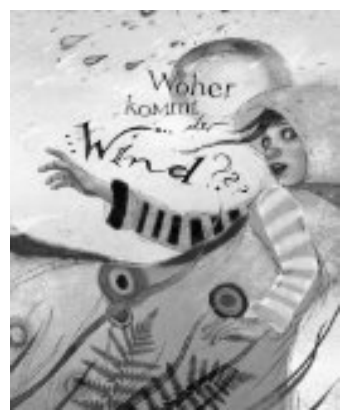

Die Mikrotypographie des als Abb. 7 präsentierten Einbandes beweist eindeutig, dass der literarische Titel Woher kommt der Wind und seine visuelle Umgebung eine Sehfläche bilden. Sowohl die dabei präsentierte Person als auch die Gestaltung des Schriftbildes knüpfen visuell an windiges Wetter an, indem sie sich nach rechts beugen. Nicht die abgebildete Person, sondern 
eben ein Teil des Titels, nämlich das Wort Wind, steht in der Mitte dieser Sehfläche. Es ist nicht nur zentriert, sondern auch gebeugt, also graphisch so stilisiert, als ob es wirklich vom Wind getragen würde. Dank dieser stilisierten Mikrotypographie werden bei den Rezipienten Assoziationen mit windigem Wetter erweckt.

Abb. 8: Recheis, Käthe: Wie das Kaninchen mutig wurde

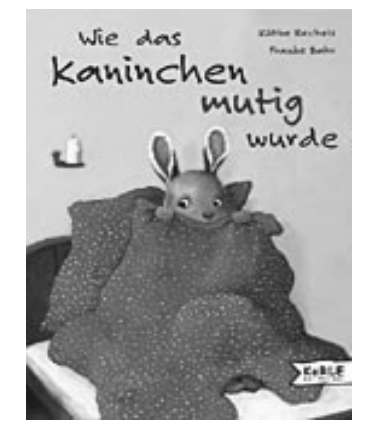

Ein anderes Beispiel dafür, wie die Sehfläche mikrotypographisch gestaltet wird, bietet Abb. 8. Die in dieser Abbildung präsentierte Sehfläche teilt sich zwar in zwei ,souveräne‘ Teilflächen (Schrift- bzw. Titelfläche und Bildfläche), aber sie teilen die Submodalität Farbe. Die im Titel dominierende Schriftfarbe ist blau, genauso wie die Decke, die auf der Bildfläche präsentiert wird und unter der das im Titel genannte Kaninchen steckt. In diesem Fall kommt es aber zu einer Art Kontradiktion (vgl. NÖTH 2000:493): Der Titel und seine visuelle Umgebung scheinen gegensätzlich zu wirken, weil das Bild dem Titel widerspricht. Auf dieser Sehfläche gehören Text und Bild nicht zum selben Zeitpunkt. Während die Bildfläche noch das ängstliche Kaninchen darstellt, wird auf der Schriftfläche schon über das mutige Kaninchen gesprochen. Die beiden Teilflächen teilen zwar die Submodalität Farbe, aber es gibt eine Ausnahme: Das einzige Wort der Schriftfläche, das rot ist, knüpft nicht an Ängstlichkeit, sondern an den Mut des Kaninchens an. So wird diese neue Eigenschaft sprachlich thematisiert und visuell präsentiert (die rote Schriftfarbe gilt als metaphorisches Lexem für Mut). 
Abb. 9: Stansbie, Stephanie: Was knarrt und raschelt in der Nacht?

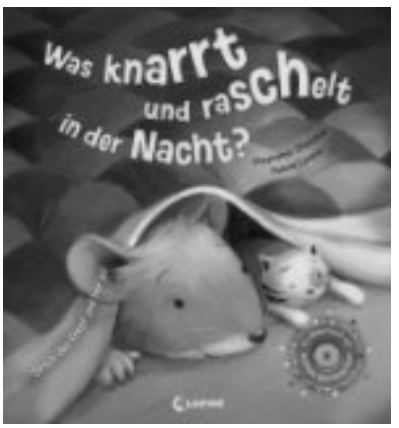

Auch die in Abb. 9 präsentierte Sehfläche verfügt auf der Teilfläche ,Titel` über eine Mikrotypographie, die an die Teilfläche ,Bild‘ visuell anknüpft. Die Gestaltung des ,gewellten“ Schriftbildes scheint die Form der auf der Teilfläche ,Bild‘ präsentierten Decke nachzuahmen. Außerdem werden einige Elemente des Titels mikrotypographisch hervorgehoben, was dank der unterschiedlichen Schriftgröße möglich ist.

Abb. 10: Dumont, Jean-François: Die kleine Gans, die aus der Reihe tanzt

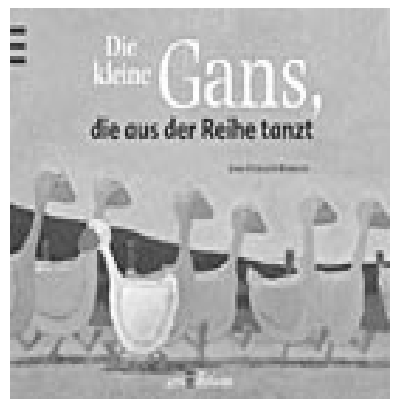

Die weiße Schriftfarbe des ersten Titelteils (Abb. 10) stimmt mit der Farbe der im Titel genannten Gans überein. Die blaue Schriftfarbe des zweiten Titelteils ist genauso wie die Farbe der Gänse, die auf dem Bild eine Reihe bilden. So wird eine Brücke gebildet, und zwar die Brücke zwischen dem sprachlichen und bildlichen Teil dieser Sehfläche.

Der Vorteil von Text-Bild-Gefügen scheint darin zu liegen, dass in verbalgraphischen Verflechtungen die Informationen sowohl sprachlich als auch bildlich dargestellt werden können. Dabei ist allerdings zu beachten, welche 
Informationen graphisch und welche verbal vermittelt werden sollten. NöTH (2000:491) nennt verschiedene Informationstypen und ordnet sie dem sprachlichen bzw. visuellen Code zu:

- Raum und Zeit: Während Bilder besonders gut geeignet dafür sind, räumlich-visuelle Zusammenhänge darzustellen, werden mit Hilfe von sprachlichen Texten eher Zeitpunkte, -räume und -verläufe thematisiert (Abb. 11).

Abb. 11: Bach, Johanna: Heute bin ich motzig und morgen wieder froh!

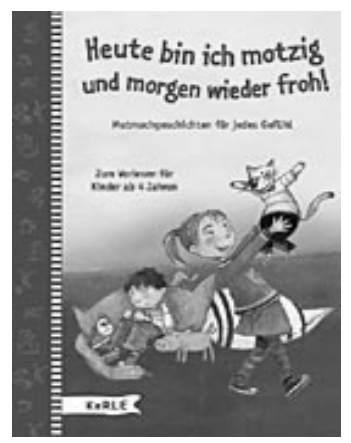

- Visuelles und Nichtvisuelles: Während alle Bilder sich beschreiben lassen, lässt sich nicht alles, was sprachlich ist, bildlich darstellen.

- $\quad$ Konkret - Abstrakt: Sprache eignet sich gut, sowohl Konkreta als auch Abstrakta zu beschreiben. Im Unterschied dazu lassen sich Abstrakta durch Bilder nur indirekt darstellen (Abb. 12).

Abb. 12: Browne, Anthony: Matti macht sich Sorgen

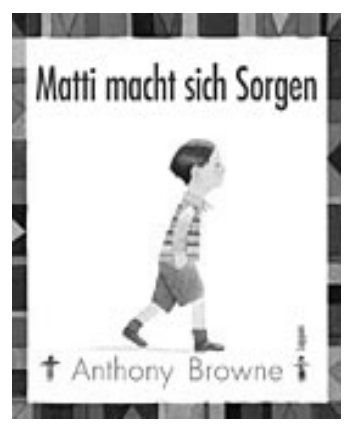

- Negation, Affirmation, Kausalität: Sowohl kausale als auch andere Beziehungen sind nur sprachlich auszudrücken (Abb. 13-14). 
Magdalena Makowska

Abb. 13: Abedi, Isabel: Leila Schwein schreit NEIN!

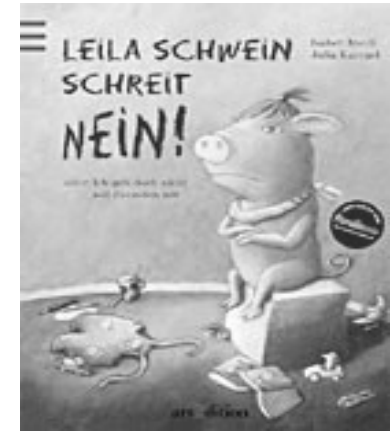

Abb. 14: Wilhelmi, Friederike: Warum schmeckt das Meer nach Salz?

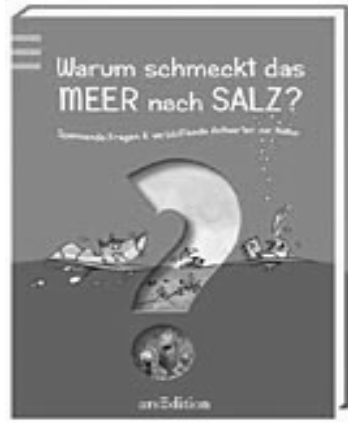

- Informationsmenge: Bilder sind in diesem Sinne informativer, denn mit ihrer Hilfe lassen sich - in der gleichen Wahrnehmungszeit mehr Informationen vermitteln.

Schon diese Beispiele beweisen eindeutig, dass Text-Bild-Beziehungen komplementär sein können: Das, was einem Element fehlt, kann durch ein anderes ergänzt werden. Im Kontext der Informativität von solchen sprachlichbildlichen Gefügen scheint es interessant zu sein, die Frage danach zu stellen, wie die Teilfläche ,Titel` nicht nur graphisch gestaltet, sondern auch formuliert wird und ob es möglich wäre, auf bestimmte syntaktisch-morphologische Gemeinsamkeiten dieser Fläche zu verweisen.

\section{Wie werden die Titel von Kinderbüchern am häufigsten for- muliert?}

Literarische Titel haben meistens die Form von kurzen Texten, die sich leicht merken und erinnern lassen. Diese kurze Form ist auch dann von Bedeutung, wenn Titel auf bebilderten Einbänden erscheinen. Auf einer solchen Sehfläche - ähnlich wie auf anderen Sehflächen, die die Menschen im Alltag begleiten - gibt es meistens nicht viel Platz für längere sprachliche Texte. Solche Sehflächen setzen sich zum Ziel, Aufmerksamkeit auf sich zu ziehen, größte Informationsmengen auf kleinstem Raum zu ermöglichen, vielfältige Präsentations-, Strukturierungs-, Orientierungs- und Rezeptionsweisen zu erlauben und den schnellen Blick zu unterstützen (vgl. SCHMITz 2011a:3). Das, was mit Worten ausgedrückt werden kann, wird auch sprachlich formu- 
liert. Und das, was sich mit Worten nur schwierig oder gar nicht beschreiben lässt, wird meistens graphisch präsentiert. Die Verknüpfung verschiedener Zeichensysteme verursacht (vgl. STÖCKL 2004:102), dass es viel leichter wird, die Schwächen einer Modalität zu bewältigen. STÖCKL (2006:18) weist darauf hin, dass ,jede Modalität [...] ihre spezifischen Codeeigenschaften [hat], die sie in der Kommunikation zu bestimmten Leistungen befähigen“. Auch in diesem Sinn gilt diese Verknüpfung verschiedener Codes als praktisch und dient der Ökonomisierung der Wahrnehmung, weil das, was der eine Code nicht schafft, der andere erreichen kann. So ist das Wesen von sprachlich-bildlichen Gefügen, denen die Forderung nach Ökonomie in der Kommunikation zugrunde liegt. In Anlehnung an SCHMITZ (2004:72) lässt sich feststellen, dass die Sprache in sprachlich-bildlichen Gefügen elliptischer als in rein schriftsprachlichen Texten sein kann, weil die Kohärenz aus dem Zusammenspiel von mehreren Zeichenressourcen erschlossen wird: „Je gewichtiger visuelle Bestandteile wirken und je stärker sie Sinnhorizonte für Texte eröffnen und festlegen, desto elliptischer kann die Sprache sein und ist es meist auch [...].“ Im Kontext von literarischen Texten - zu denen Kinderbücher und ihre Titel gehören - weist FIX (1998:166f.) darauf hin, dass „ein Text mit unvollständiger Kohäsion [...] allein schon durch seine unverbunden nebeneinander stehenden Wörter ein gewisses Maß an Kohäsion aufweisen [könnte]“. Die Autorin vertritt die Meinung, dass im Falle von grammatikarmen Texten „Bedeutung für Syntax ein[springt]“. Die Analyse von literarischen Titeln, die auf bebilderten Kinderbucheinbänden auftauchen, hat eindeutig bewiesen, dass auch „im Wort bereits aktivierende Grammatik steckt“. In diesem Fall kommt es oft vor, dass der literarische Titel ein Bedeutungspotenzial gibt, das durch einen entsprechenden Kontext aktiviert und erschlossen werden muss. Einen solchen Kontext sichert häufig die visuelle Umgebung des Titels, die zusammen mit diesem Titel eine Sehfläche bildet. 
Abb. 15: Abedi, Isabel: Wie lange noch?

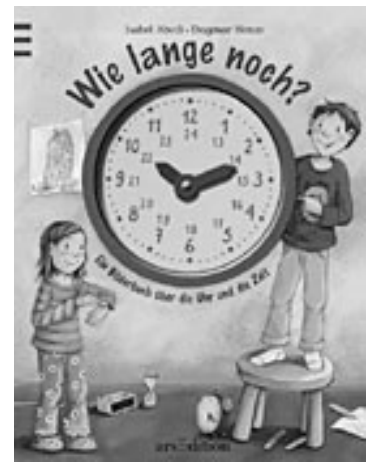

Der Titel des in Abb. 15 präsentierten Einbandes hat die Form einer elliptischen Frage. Ohne die visuelle Begleitung könnte das Bedeutungspotenzial, das dieser elliptische Titel mit sich bringt, unterschiedliche Assoziationen erwecken, wie z.B.:

a. Wie lange noch [bleibst du hier]?

b. Wie lange noch [muss ich auf dich warten]?

c. Wie lange noch [dauert das]?

Obwohl dieser elliptische Titel zweifelsohne zu einem grammatikarmen Text wird, vermittelt er ein Bedeutungspotenzial und dieses wird dank dem bildlichen Kontext erweitert. Dank der Abbildung der Uhr, die auf der Teilfläche ,Bild‘ auftaucht, kann vermutet werden, dass das Buch die Frage der Uhrzeit thematisiert.

Die linguistische Analyse von Kinderbuchtiteln hat bestätigt, dass sich im Fall von solchen Titeln einige morphologisch-syntaktische Regelmäßigkeiten feststellen lassen (vgl. MAKOWSKA 2012). Wenn der Klassifikation von Titeltypen, Titelformen und Titelmustern gefolgt wird, die von NoRD (1993) vorgeschlagen wurde, lässt sich beobachten, dass den am häufigsten vorkommenden Typ die Einfachtitel bilden (89,5\% der analysierten Titeleinheiten). Aufgrund dessen scheint es völlig legitim zu sein, das untersuchte Korpus als Einfachtitelkorpus zu betrachten. Unter den Einfachtiteln lassen sich folgende Varianten feststellen:

a. Ein-Nomen-Titel, z. B.: Dulleck, Nina: Hüpfer;

b. Nominalphrase, z. B.: Battut, Eric: Der rote Max; 
c. Kombination von Nominalphrasen, z.B.: Baumgart, Klaus: Lauras Stern und die Traummonster;

d. satzförmige Titel, z.B.: Doinet, Mymi: Leo hat eine feuerrote Brille; Funke, Cornelia: Wo das Glück wächst.

Die am häufigsten vorkommende Titelform (57,5\% der analysierten Titeleinheiten) bilden nominale Titel, die meistens als Eigennamen oder Appellativa mit oder ohne Ergänzung erscheinen (vgl. MAKOWSKA 2012). Dabei werden Titelmuster repräsentiert, deren Kern sowohl nicht erweiterte (z.B. Gréban, Quentin: Pünktchen) als auch mit Possessivpronomina, Artikeln oder Adjektiven erweiterte Nomen bilden (z.B. Janisch, Heinz: Die Brücke; Mueller, Dagmar H.: Die unsichtbare Noa; Baethmann, Teresa: Mein großes Naturbuch). Nomen mit und ohne Erweiterungen werden als Nominalphrase ${ }_{1}$ oder - wenn dabei Konjunktionen vorkommen (was aber nicht obligatorisch ist) als Kombination von mehreren Nominalphrasen (Nominalphrase ${ }_{1}+$ (Konjunktion) + Nominalphrase $_{n}$ bzw. Erweiterung) bezeichnet (z.B. NP - NP: Bartram, Angelika: Kleine Helden - großer Mut; NP + (Konjunktion) + NP: Cowell, Cressida: Kathi Braun und das Ungeheuer; NP + Relativsatz als Erweiterung: Dumont, Jean-François: Die kleine Gans, die aus der Reihe tanzt).

Die linguistische Analyse von literarischen Titeln beweist, dass die meisten nominalen Einfachtitel Kombinationen von Nominalphrasen sind (45\% aller nominalen Einfachtitel). Ihnen folgen Titeleinheiten, die aus Nomen mit dem bestimmten Artikel und Adjektiv bestehen (15\%). Verhältnismäßig häufig kommen aber auch solche Titeleinheiten vor, in denen reine Nomen (12\%) oder Nomen mit (voran- oder nachgestelltem) Genitivattribut (11\%) auftreten (z.B. Bolliger-Savelli, Antonella: Gackitas Ei; Cortazar, Julio: Rede des Bären).

Aus der syntaktischen Analyse geht hervor, dass diesen nominalen Titeln von Kinderbüchern Titel folgen, die 34\% aller Einfachtitel ausmachen. Darunter lassen sich die folgenden Titelmuster unterscheiden:

- $\quad$ satzförmiges Titelmuster 1 (Sm1; 56\%): Aussagesatz Doucet, Sharon Arms: Lucy rettet Mama Kroko

- $\quad$ satzförmiges Titelmuster 2 (Sm2; 12,5\%): Imperativsatz Böse, Susanne: Summ, kleine Biene!

- $\quad$ satzförmiges Titelmuster 3 (Sm3; 15,5\%): direkter Fragesatz Kämpf, Christian: Wer schläft denn da? 
Magdalena Makowska

- $\quad$ satzförmiges Titelmuster 4 (Sm4; 7\%): indirekter Fragesatz Hercíková, Iva: Wie das Hündchen Junge haben wollte

- $\quad$ satzförmiges Titelmuster ${ }_{5}$ (Sm5; 3,5\%): Temporalsatz

Nilsson, Ulf: Als Oma seltsam wurde

- $\quad$ satzförmiges Titelmuster 6 (Sm6; 3\%): Hauptsatz-, NebensatzGefüge

Bosch, Pseudonymous: Wenn du dieses Buch liest, ist alles zu spät

Unter den satzförmigen Titeleinheiten sind die Aussagesätze mit 56\% am häufigsten repräsentiert. Ihnen folgen die Fragesätze, die 15,5\% des Korpus ausmachen, gefolgt von Imperativsätzen, die 12,5\% der satzförmigen Titel von Kinderbüchern bilden.

Es steht außer Zweifel, dass alle präsentierten Titelformen und -muster den Kindern gut bekannt sein können, weil sie in alltäglichen Kinder-ElternInteraktionen zuerst nachgeahmt und dann ganz bewusst gebraucht werden. Wenn diese grammatischen Strukturen im Fall von bebilderten Kinderbucheinbänden in einen grafischen Kontext eingebettet sind, werden sie von Kindern als eine komplexe Sehfläche spontan wahrgenommen. Deshalb scheint es interessant zu sein, im Fall von Kinderbucheinbänden auch danach zu fragen, ob solche Sehflächen den kindlichen Spracherwerb beeinflussen könnten.

\section{Ist es möglich, mit Hilfe von Kinderbucheinbänden den kind- lichen Spracherwerb zu fördern?}

Die oben präsentierte Analyse hat bewiesen, dass sich im Fall von analysierten Titeln bestimmte morphologisch-syntaktische Regelmäßigkeiten feststellen lassen. Die meisten von diesen Titeln sind Einfachtitel, die der Form nach nominale oder satzförmige Titeleinheiten bilden. Unter den nominalen Einfachtiteln sind am häufigsten diejenigen repräsentiert, die dem Titelmuster Nominalphrase + Nominalphrase folgen. Das lässt sich damit erklären, dass die meisten Kinderbücher ihre Protagonisten schon im Titel präsentieren, wenn z. B. Namen von Protagonisten genannt werden (Abb. 16-17). 
Abb. 16: Sauermann, Marcus: Der Kleine und das Biest

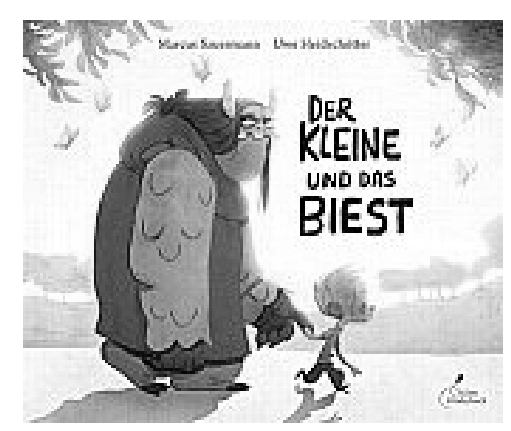

Abb. 17: Strid, Jakob Martin: Die unglaubliche Geschichte von der Riesenbirne

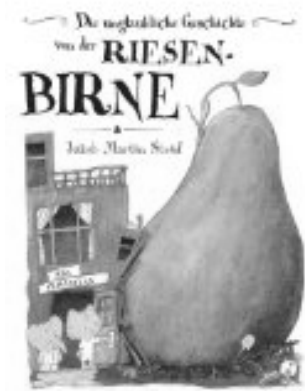

Auch satzförmige Titelkonstruktionen sind Kindern gut bekannt, weil schon ganz kleine Kinder von ihren Eltern eben mit solchen Sätzen in der ,Motherese` am häufigsten angesprochen werden. ${ }^{6}$ Die Analyse hat bewiesen, dass die Sprache, die in Titeln von Kinderbüchern gebraucht wird, eben an ,Motherese“ als natürliche Sprache anknüpft. Unter ,Motherese“ wird eine Sprechweise verstanden, die die Eltern gebrauchen, wenn sie ihre Kleinkinder ansprechen. Einerseits ist sie hoch intuitiv, andererseits aber ist sie durch besondere Merkmale gekennzeichnet: Nachahmung, viele Fragen, Hier-undJetzt-Gespräche, syntaktische Einfachheit, die Aufmerksamkeit erzeugende Prosodie und Intonation oder ein vermindertes Sprechtempo. Diese Elemente der ,Motherese‘ lassen sich in vielen Titeln von Kinderbüchern finden:

In Anlehnung an ZoLlinger (2008:48f.) wird die Sprache, die ein Erwachsener mit einem Baby in einer besonderen Art und Weise spricht, als ,Motherese“ bezeichnet. 
Abb. 18: Geisler, Dagmar: Plitsch, platsch, Badespaß!

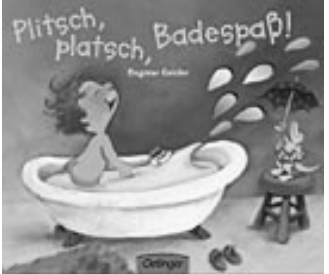

Abb. 21: Senner, Katja \& Grimm, Sandra: Hoppla, Paulchen!

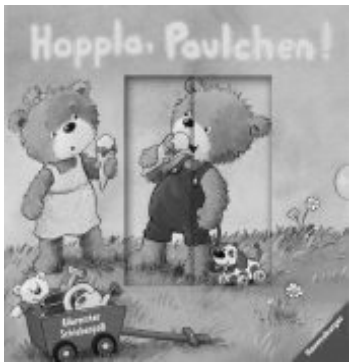

Abb. 19: Hansen, Christiane \& Grimm, Sandra: Bist du meine Mama?

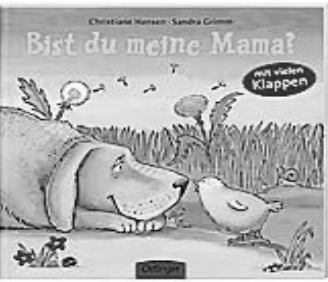

Abb. 22: Kraushaar, Sabine: Soooo müde!

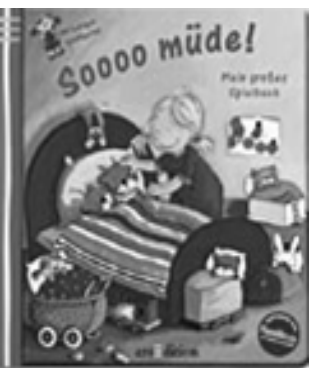

Abb. 20: Schwarz, Regina: Schnullerlos! Ich bin schon groß!

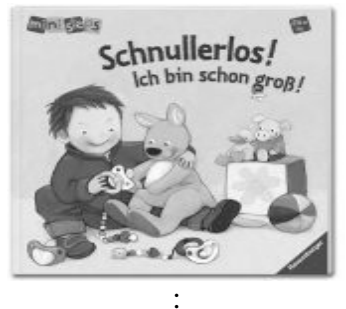

Abb. 23: Benthin, Anna Dein Engel hat dich gern

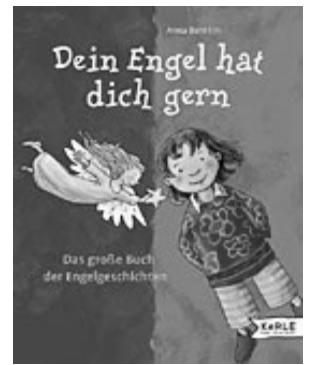

Der in Abb. 18 präsentierte Einband knüpft sprachlich (plitsch, platsch) und bildlich (ein Kind in der Badewanne) an das den Kindern bekannte Format ,baden' an, d.h. an eine Situation, mit der auch Kleinkinder gut vertraut sind. Der Titel des Buches Bist du meine Mama? hat die Form einer Frage. Fragen gelten als Konstruktionen, die in die Interaktion einbeziehen und dadurch für die Entwicklung der Sprachkompetenz besonders relevant sind. Auch der in Abb. 20 präsentierte Bucheinband thematisiert, was den Kindern aus dem Alltag bekannt ist, und zwar die Schnuller-Frage. Das Buch Hoppla, Paulchen! macht vom Wort hoppla Gebrauch, und auch dieses Wort erscheint ganz oft in Eltern-Kind-Interaktionen (als Anregung zum gemeinsamen Spielen). Die Struktur des Titels Soooo müde ähnelt dem verminderten Sprechtempo von Eltern, was der Aufmerksamkeitszentrierung dient. Der letzte der präsentierten Kinderbucheinbände (Dein Engel hat dich gern) spricht das Kind sowohl verbal (das Possessivpronomen dein und das Personalpronomen 
dich), als auch visuell an (das Gesicht in der frontalen Perspektive hat einen auffordernden Charakter).

\section{Fazit}

Zusammenfassend lässt sich feststellen, dass bebilderte Kinderbucheinbände zweifelsohne zu komplexen Sehflächen werden, auf denen sprachliche und bildliche Elemente synergetisch zusammenwirken. Kinderbucheinbände als Sehflächen werden in einem Kognitionsakt holistisch wahrgenommen und funktional interpretiert. Mit Hilfe von so gestalteten Sehflächen ist es möglich, Kinder als Rezipienten anzusprechen, ihre Blicke zu fangen und ihnen Informationen mitzuteilen. Die ,Titelsprache', die formal und semantisch der ,Motherese` ähnelt, verursacht, dass mit Hilfe von Titeln und ihrer visuellen Umgebung der kindliche Spracherwerb gefördert werden kann. Die ,Motherese', die in literarischen Titeln wiederkehrt, hat nicht nur eine kommunikative Funktion, sondern auch eine Sprachlernfunktion zu erfüllen. Die Mischung von sprachlichen und bildlichen Elementen auf einer Sehfläche folgt den Möglichkeiten des menschlichen bzw. kindlichen Gehirns und richtet sich nach Regeln, dank denen es möglich ist, das Zusammenspiel beider Hemisphären zu unterstützen. Bebilderte Kinderbucheinbände, die zu komplexen Sehflächen werden, thematisieren meistens Formate, die in Beziehung zur Erfahrungswelt der Kinder stehen. Diese Formate werden erkannt und damit werden auch erste Transferleistungen erbracht. Der Umgang mit solchen Sehflächen gilt auch als Schlüsselsituation, in der Kommunikation über ein Drittes, d.h. über ein Buch, vermittelt wird. Das hat zur Folge, dass bebilderte Kinderbucheinbände, die als Brücke zu richtigen, literarischen Kotexten dienen, aktivierend wirken können. Der Spracherwerb erfolgt durch die selbstorganisierte Auseinandersetzung des Kindes mit der Umwelt. Den Anlass dazu bilden bebilderte Kinderbucheinbände, die Kinder verbal und graphisch ansprechen und zum Sprechen motivieren. 
Magdalena Makowska

\section{Literatur}

BEAUgRANDE De, RoBert-Alain / DRessler, WolfGang UlRICH (1981): Einführung in die Textlinguistik. Tübingen (=Konzepte der Sprach- und Literaturwissenschaft 28). BuCHER, HANs-JüRGEN (1996): Textdesign - Zaubermittel der Verständlichkeit? Die Tageszeitung auf dem Weg zum interaktiven Medium. In: Hess-LÜTTICH, ERNEST W. B. / Holly, WeRner / PÜsChel, UlRich (eds.): Textstrukturen im Medienwandel. Frankfurt (M.)/Berlin/Bern u. a., 31-59.

- (2000): Formulieren oder Visualisieren? Multimodalität in der Medienkommunikation. In: Richter, GERD / RIECKE, JÖRG / SCHUSTER, BRITT-MARIE (eds.): Raum, Zeit, Medium - Sprache und ihre Determinanten. Darmstadt, 661-691.

- (2011): Multimodales Verstehen oder Rezeption als Interaktion. Theoretische und empirische Grundlagen einer systematischen Analyse der Multimodalität. In: DIEKMANNSHENKE / KLEMM / STÖCKL, 123-156.

Burger, Harald ( $\left.{ }^{3} 2005\right):$ Mediensprache. Eine Einführung in Sprache und Kommunikationsformen der Massenmedien. Berlin.

Diekmannshenke, Hajo / Klemm, Michael / StÖcKL, Hartmut (eds.) (2011): Bildlinguistik. Theorie - Methoden - Fallbeispiele. Berlin.

EHLICH, KonRAD (2005): Sind Bilder Texte? In: Der Deutschunterricht 4:51-60.

Feilke, Helmuth / Linke, Angelika (eds.) (2009): Oberfläche und Performanz. Untersuchungen zur Sprache als dynamischer Gestalt. Tübingen.

FIX, Ulla (1998): Die Wörter auf dem Papier und die Grammatik in den Köpfen. Zur Textualität und zu Lesearten von ,grammatikarmen“ Texten. In: BARZ, IRMHILD / ÖHLSCHLÄGER, GÜNTHER (eds.): Zwischen Grammatik und Lexikon. Tübingen, 165-177.

- (2001): Grundzüge der Textlinguistik. In: Fleischer, Wolfgang / HelBIG, GERHARD / LERCHNER, GOTTHARD (eds.): Kleine Enzyklopädie - deutsche Sprache. Frankfurt (M.)/Berlin/Bern u. a., 470-511.

- (2008): Text und Textlinguistik. In: JANICH, NinA (ed.): Textlinguistik. 15 Einführungen. Tübingen, 15-34.

- (2009): Aktuelle linguistische Textbegriffe und der literarische Text. Bezüge und Abgrenzungen. In: WinKo, SimONE / JANNIDIS, Fotis / LAUER, GERHARD (eds.): Grenzen der Literatur: Zu Begriff und Phänomen des literarischen. Berlin, 103-135.

Greimas, AlgiRdas (1971): Strukturelle Semantik. Braunschweig.

Hess-LÜTTICH, ERNEST W.B. (1997): Text, Intertext, Hypertext. Zur Texttheorie der Hypertextualität. In: KLEIN, JOSEF / FIX, UlLA (eds.): Textbeziehungen. Linguistische und literaturwissenschaftliche Beiträge zur Intertextualität. Tübingen, 125-148

Holly, WeRNer (2009): Der Wort-Bild-Reißverschluss. Über die performative Dynamik der audiovisuellen Transkriptivität. In: FEILKE / LINKE, 389-406. 
JÄGER, LUDWIG (2002): Transkriptivität. Zur medialen Logik der kulturellen Semantik. In: JÄGER, LUDWIG / STANITZEK, GEORG (eds.): Transkribieren. Medien/Lektüre. München, 19-41.

JANICH, NiNA ( $\left.{ }^{4} 2005\right):$ Werbesprache: ein Arbeitsbuch. Tübingen.

Klemm, Michael / StÖCKL, HARTMUT (2011): Bildlinguistik - Standortbestimmung, Überblick, Forschungsdesiderate. In: DIEKMANNSHENKE / KLEMM / STÖCKL, 7-18.

KrÄMER, SyBILlE (2006): Die Schrift als Hybrid aus Sprache und Bild. Thesen über die Schriftbildlichkeit unter Berücksichtigung von Diagrammatik und Kartographie. In: Hoffmann, ToRsten / RipPL, Gabriele (eds.): Bilder. Ein (neues) Leitmedium? Göttingen, 79-92.

Kress, Gunther / VAN LEEUWen, THeO (1996): Reading images. The grammar of visual design. London.

Makowska, Magdalena (2012): (Mikro)Syntax in Mikrotexten. Syntaktische Analyse von Märchentiteln. In: Acta Neophilologica XIV(2):69-78.

NÖTH, WinfRIED (2000): Der Zusammenhang von Text und Bild. In: BRINKER, KLAUS / Antos, Gerd / Heinemann, Wolfgang / SAger, Sven (eds.): Text- und Gesprächslinguistik. HSK-Bd. 16.1. Berlin/New York, 489-496.

NoRD, CHRISTIANE (1993): Einführung in das funktionale Übersetzen. Am Beispiel von Titeln und Überschriften. Tübingen.

SAndig, BARBARA (2000): Textmerkmale und Sprache-Bild-Text. In: FIX, Ulla / Wellmann, Hans (eds.): Bild im Text - Text im Bild. Heidelberg, 3-30.

Schmitz, UlRICH (2001): Stets heikle Kohärenz in Text-Bild-Gefügen. Sinnsuche auf Papier und Sinnkonstruktion am Computer. In: Hess-LÜtTICH, ERNEST W. B. (ed.): Medien, Texte und Maschinen. Wiesbaden, 141-165.

- (2003): Lesebilder im Internet. Neue Koalitionen und Metamorphosen zwischen Text und Bild. In: Zeitschrift für Germanistik. Neue Folge XIII/3:605-628.

- (2003a): Text - Bild-Metamorphosen in Medien um 2000. In: SCHMITZ, UlRich (ed.): Wissen und neue Medien. Bilder und Zeichen von 800 bis 2000. Berlin, 241-263.

- (2004): Schrift und Bild im öffentlichen Raum. In: Mitteilungen des Deutschen Germanistenverbandes 1:58-74.

- (2005): Blind für Bilder. Warum sogar Sprachwissenschaftler auch Bilder betrachten müssen. In: Osnabrücker Beiträge zur Sprachtheorie 69:187-227.

- (2011): Blickfang und Mitteilung. Zur Arbeitsteilung von Design und Grammatik in der Werbekommunikation. In: Zeitschrift für Angewandte Linguistik (ZfAL) 54:79-109.

- (2011a): Sehflächenforschung. Eine Einführung. In: DieKMANNSHENKE / KLEMM / STÖCKL, 1-20.

SCHNEIDER, JAN GEORG / STÖCKL, HARTMUT (2011): Medientheorien und Multimodalität: Zur Einführung. In: SCHNEIDER, JAN GEORG / STÖCKL, HARTMUT (eds.): Medien- 
theorien und Multimodalität. Ein TV-Werbespot - Sieben methodische Beschreibungsansätze. Köln, 10-38.

Scholz, Oliver (1998): Was heißt es, ein Bild zu verstehen? In: SACHs-Hombach, KlAUS / REBKÄMPER, KlAus (eds.): Bild - Bildwahrnehmung - Bildverarbeitung. Interdisziplinäre Beiträge zur Bildwissenschaft. Wiesbaden, 105-117.

SPITZMÜLLER, JÜRGEN (2009). Typographisches Wissen: die Oberfläche als semiotische Ressource. In: FEILKE / LINKE, 459-486.

STÖCKL, HARTMUT (1997): Werbung in Wort und Bild. Textstil und Semiotik englischsprachiger Anzeigenwerbung. Frankfurt (M.)/Berlin.

- (2004): Bilder - Konstitutive Teile sprachlicher Texte und Bausteine zum Textstil. In: Mitteilungen des Deutschen Germanistenverbandes 2:102-120.

- (2004a): Die Sprache im Bild - das Bild in der Sprache. Zur Verknüpfung von Sprache und Bild im massenmedialen Text. Konzepte, Theorien, Analysemethoden. New York/Berlin.

- (2004b): Typographie: Gewand und Körper des Textes - Linguistische Überlegungen zu typographischer Gestaltung. In: Zeitschrift für Angewandte Linguistik 41:5-48.

- (2005): Anschauungsorientierung im Text - Zwischen Sprache und Bild. In: FiX, Ulla / Lerchner, GotTHARd / SchröDer, Marianne / Wellmann, Hans (eds.): Zwischen Lexikon und Text - Lexikalische, stilistische und textlinguistische Aspekte. Stuttgart/Leipzig, 64-79.

- (2006): Zeichen, Text und Sinn - Theorie und Praxis der multimodalen Textanalyse. In: EcKKrammer, Eva MARIE / Held, GudRun (eds.): Textsemiotik. Studien zu multimodalen Medientexten. Frankfurt (M.)/Berlin/Bern u. a., 11-26.

- (2011): Sprache-Bild-Texte lesen. Bausteine zur Methodik einer Grundkompetenz. In: DieKMANNSHENKE / KLEMm / STÖCKL, 43-70.

STORRER, ANGELIKA (2004): Text-Bild-Bezüge und Nutzermetaphern im World Wide Web. In: Mitteilungen des Deutschen Germanistenverbandes 1:40-57.

Wolf, Norbert R. (2000): Texte als Bilder. In: Fix, Ulla / Wellmann, Hans (eds.): Bild im Text - Text im Bild. Heidelberg (=Sprache - Literatur und Geschichte 20), 289-305.

ZOLLINGER, BARBARA ( $\left.{ }^{8} 2008\right):$ Spracherwerbsstörungen. Grundlagen zur Früherfassung und Frühtherapie. Bern/Stuttgart/Wien.

\section{Literatur auf Websites}

KRÄMER, SYBILLE (2005): ,Operationsraum Schrift‘. Über einen Perspektivenwechsel in der Betrachtung der Schrift. (19.11.2012).

http://userpage.fu-berlin.de/ sybkram/media/downloads/Operationsraum_Schrift.pdf 